\title{
Factors Affecting Contrast Sensitivity in Intermittent Exotropia
}

\author{
Ji Min Kwon, Soo Jung Lee \\ Department of Ophthalmology, Haeundae Paik Hospital, Inje University College of Medicine, Busan, Korea
}

Purpose: To determine whether contrast sensitivity (CS) can represent photophobia in intermittent exotropia (IXT) by comparing the CS test with and without glare stimulus, and to analyze the factors of IXT affecting CS.

Methods: We retrospectively reviewed the medical records of 107 patients with basic-type IXT. We compared CS under mesopic and photopic conditions, with and without glare. We compared the difference in CS before and after glare ( $\Delta C S)$ between mesopic and photopic conditions, and compared CS with glare between patients with and without photophobia. The correlations between the clinical features of IXT and CS were analyzed.

Results: There was no significant reduction in CS at all spatial frequencies by glare stimulus. $\Delta$ CS was greater at high spatial frequencies under photopic conditions than under mesopic conditions. The group with subjective photophobia showed lower CS at $10.2 \mathrm{cpd}$ under mesopic conditions with glare. CS showed a negative correlation with stereopsis under both mesopic and photopic conditions, and a positive correlation with fusional ability at low and intermediate spatial frequencies under mesopic conditions. $\Delta \mathrm{CS}$ was smaller at intermediate spatial frequencies with better fusional ability, greater at high spatial frequencies with photophobia, and greater at intermediate spatial frequencies with a higher frequency of exotropia.

Conclusions: The CS test could not represent photophobia in IXT. However, CS tended to decrease with glare stimulus, and CS under mesopic conditions with glare was worse when accompanied by photophobia. Moreover, a poorer degree of stereopsis was associated with lower CS, and better fusional ability was associated with higher CS under mesopic conditions. Therefore, the CS test can be considered helpful in evaluating sensory function in IXT.

Key Words: Contrast sensitivity, Depth perception, Exotropia, Photophobia

Intermittent exotropia (IXT) is the most common type of exotropia and is typically accompanied by subjective symptoms, with the most common being photophobia. Wang and Chryssanthou [1] reported that photophobia was elicited in $76 \%$ of patients presenting with symptoms of IXT. Oh et al. [2] reported that $60 \%$ of patients with IXT

Received: April 23, 2020 Final revision: July 28, 2020

Accepted: July 30, 2020

Corresponding Author: Soo Jung Lee, MD, PhD. Department of Ophthalmology, Haeundae Paik Hospital, Inje University College of Medicine, 875 Haeundae-ro, Haeundae-gu, Busan 48108, Korea. Tel: 82-51797-2310, Fax: 82-51-797-2321, E-mail: kris9352@paik.ac.kr had subjective symptoms and that $90 \%$ of them demonstrated photophobia. The mechanism underlying photophobia in exotropia remains unclear. It has been believed that bright light stimulates the retina, reduces fusional function, and induces manifest strabismus, and photophobia appears to prevent the resulting diplopia and visual confusion [3]. However, there is currently no clear tool to objectively evaluate subjective symptoms such as photophobia. Some studies have evaluated photophobia by using contrast sensitivity (CS) measurement, which assesses the reduction in visual function after exposure to a strong light stimulus with specific illuminance. CS refers to the ability 
to detect a difference in brightness (or luminance) between adjacent objects or areas in a space. CS may be abnormal in amblyopia, optic neuritis, cataract, and glaucoma, but also decreases in the presence of photophobia [4,5]. Lew et al. [6] reported that photophobia in IXT was associated with a distant exodeviation greater than 25 prism diopters (PD) and stereoacuity worse than 60 arcsec, but no analysis was performed with CS measurement. Chung et al. [7] observed that CS under mesopic conditions could be indicative of photophobia in children with IXT. However, there was no correlation between a significant decrease in CS with glare and angle of deviation, sex, age, spherical equivalent (SE), stereopsis, binocular status, and duration of IXT.

We wondered whether CS could represent photophobia in IXT, and tried to determine if other factors besides photophobia affected CS. Therefore, we conducted CS tests in patients with IXT and investigated whether CS decreased significantly by glare stimuli in IXT, whether CS differs depending on the presence of photophobia, and analyzed the factors affecting CS measured at each visual angle.

\section{Materials and Methods}

We retrospectively analyzed the medical records of 107 patients who had been diagnosed with a basic-type IXT and were able to cooperate in CS tests between August 2017 and September 2019. Patients with a history of amblyopia, ocular abnormality, hyperopia or myopia $\geq 6$ diopters (D), astigmatism $\geq 2 \mathrm{D}$, paralytic strabismus, restrictive strabismus, previous ocular surgery including strabismus surgery, nystagmus, congenital deformity, neurologic abnormality, chromosomal disorder, or systemic diseases were excluded.

Sex, age at diagnosis, best-corrected visual acuity (BCVA, logarithm of the minimum angle of resolution), SE, angle of deviation, stereopsis, binocular status, and CS were recorded. Age of onset, frequency of IXT, and subjective photophobia assessments were also collected by interviewing patients and their caregivers. Considering the nature of IXT with many children, the symptoms of subjective photophobia were not limited to the patient's glare appeal, but included frequent blinking, severe frowning, and turning of the head to avoid bright lights. The angle of deviation was measured at a distance of $33 \mathrm{~cm}$ (near) and
$6 \mathrm{~m}$ (far) using an alternate prism cover test. Stereopsis was evaluated by the Titmus stereoacuity test (Stereo Optical Co., Chicago, IL, USA) at near, and the binocular status was assessed using the Worth 4 Dot test for both near and far distances.

Regarding CS, the contrast threshold was measured binocularly according to the presence or absence of glare under mesopic (average luminance of 5 candelas/square meter $\left[\mathrm{cd} / \mathrm{m}^{2}\right]$ ) and photopic conditions (average luminance of $100 \mathrm{~cd} / \mathrm{m}^{2}$ ) using the Contrast Glare tester 2000 (CGT2000; Takagi Seiko, Nagano, Japan) with refractive correction. The contrast threshold refers to the minimum contrast that a patient can see, and 8 glare lamps with a brightness of $40,000 \mathrm{~cd} / \mathrm{m}^{2}$ under mesopic conditions and 100,000 cd/ $\mathrm{m}^{2}$ under photopic conditions were given as the glare stimulus. Measurements were taken at the following six visual angles: $6.3^{\circ}, 4^{\circ}, 2.5^{\circ}, 1.6^{\circ}, 1^{\circ}$, and $0.64^{\circ}$. Visual angles were converted to cycles per degree for better understanding and comparison with other papers (each corresponds to 1.1, $1.8,2.9,4.5,7.1$, and 10.2 cycles per degree [cpd], respectively) [8]. Since the results of the tester were indicated as a contrast threshold, the values were converted to log contrast sensitivity $(\log C S)$ for statistical analysis [9]. The test proceeded in the following order: mesopic without glare, mesopic with glare, photopic without glare, and photopic with glare.

The paired $t$-test was used to analyze significant decreases in CS due to the glare at each spatial frequency, and to compare the difference in CS before and after glare stimulus $(\triangle \mathrm{CS})$ between mesopic and photopic conditions. The independent $t$-test was used to compare the CS with glare stimulus between patients with and without photophobia. The correlations between BCVA, SE, angle of deviation, stereopsis, binocular status, age of onset, frequency, subjective photophobia, and CS were analyzed using Pearson correlation analysis, followed by an analysis of the correlation between BCVA, SE, angle of deviation, stereopsis, binocular status, age of onset, frequency, subjective photophobia, and $\triangle \mathrm{CS}$. The correlations between BCVA, SE, angle of deviation, stereopsis, binocular status, age of onset, frequency, and subjective photophobia were also analyzed. The analyses were conducted using IBM SPSS Statistics ver. 22.0 (IBM Corp., Armonk, NY, USA), and $p$-values $<0.05$ were considered statistically significant.

This study was approved by the institutional review board of Inje University Haeundae Paik Hospital (2010-01- 
Table 1. Log contrast sensitivity before and after glare stimulus

\begin{tabular}{lccc}
\hline $\begin{array}{l}\text { Spatial } \\
\text { frequency (cpd) }\end{array}$ & Without glare & With glare & $p$-value \\
\hline Mesopic & & & \\
1.1 & 1.666 & 1.646 & 0.753 \\
1.8 & 1.645 & 1.577 & 0.227 \\
2.9 & 1.487 & 1.449 & 0.514 \\
4.5 & 1.229 & 1.190 & 0.489 \\
7.1 & 0.904 & 0.891 & 0.805 \\
10.2 & 0.563 & 0.564 & 0.980 \\
Photopic & & & \\
1.1 & 1.791 & 1.782 & 0.872 \\
1.8 & 1.729 & 1.758 & 0.673 \\
2.9 & 1.697 & 1.662 & 0.609 \\
4.5 & 1.490 & 1.468 & 0.755 \\
7.1 & 1.232 & 1.180 & 0.436 \\
10.2 & 0.947 & 0.870 & 0.216 \\
\hline
\end{tabular}

$\mathrm{cpd}=$ cycles per degree

Table 2. Differences in log contrast sensitivity before and after glare stimulus

\begin{tabular}{lccc}
\hline Spatial frequency $(\mathrm{cpd})$ & Mesopic & Photopic & $p$-value \\
\hline 1.1 & 0.339 & 0.267 & 0.139 \\
1.8 & 0.298 & 0.282 & 0.736 \\
2.9 & 0.229 & 0.228 & 0.973 \\
4.5 & 0.206 & 0.246 & 0.262 \\
7.1 & 0.183 & 0.253 & $0.030^{*}$ \\
10.2 & 0.186 & 0.264 & $0.014^{*}$ \\
\hline
\end{tabular}

$\mathrm{cpd}=$ cycles per degree.

${ }^{*} p<0.05$.

009-001). Written informed consent was waived due to the retrospective nature of the study.

\section{Results}

A total of 107 patients were included, with 36 boys (33.6\%) and 71 girls (66.4\%). Their mean age was $7.4 \pm 2.3$ years (range, 4 to 19 years), mean BCVA was $0.0 \pm 0.0$ (range, 0.0 to 0.1 ), and mean SE was $-1.0 \pm 1.9 \mathrm{D}$ (range, -5.75 to $+4.5 \mathrm{D})$. The mean deviation angle was $28.4 \pm 6.6$
Table 3. Log contrast sensitivity with glare stimulus in groups with and without subjective photophobia

\begin{tabular}{lccc}
\hline $\begin{array}{l}\text { Spatial } \\
\text { frequency (cpd) }\end{array}$ & $\begin{array}{c}\text { Subjective } \\
\text { photophobia }(+)\end{array}$ & $\begin{array}{c}\text { Subjective } \\
\text { photophobia }(-)\end{array}$ & $p$-value \\
\hline Mesopic & 1.641 & 1.650 & 0.907 \\
1.1 & 1.565 & 1.583 & 0.776 \\
1.8 & 1.444 & 1.452 & 0.905 \\
2.9 & 1.137 & 1.221 & 0.282 \\
4.5 & 0.827 & 0.929 & 0.220 \\
7.1 & 0.468 & 0.622 & $0.047^{*}$ \\
10.2 & & & \\
Photopic & 1.781 & 1.782 & 0.989 \\
1.1 & 1.759 & 1.758 & 0.999 \\
1.8 & 1.709 & 1.634 & 0.376 \\
2.9 & 1.495 & 1.452 & 0.615 \\
4.5 & 1.160 & 1.193 & 0.735 \\
7.1 & 0.776 & 0.926 & 0.101 \\
10.2 & & & \\
\hline
\end{tabular}

cpd $=$ cycles per degree. $\stackrel{*}{p}<0.05$.

PD (range, 14 to $45 \mathrm{PD}$ ) at distance and $30.6 \pm 6.7 \mathrm{PD}$ (range, 16 to $50 \mathrm{PD}$ ) at near. The mean stereoacuity was $95.7 \pm 132.1$ arcsec (range, 40 to 800 arcsec). In the Worth 4 Dot test, 27 patients had fusional ability at both distance and near, 10 had fusional ability only near, and 70 had no fusional ability. The mean age of onset was $4.3 \pm 2.6$ years (range, 0.6 to 11 years), and the frequency was $31.8 \pm 24.1 \%$ (range, $0 \%$ to $90 \%$ ). Among the 107 patients, 40 (37.4\%) complained of subjective photophobia.

There was no spatial frequency with a significant reduction in $\log C S$ with a glare stimulus under both mesopic and photopic conditions (Table 1). $\triangle \mathrm{CS}$ was greater under photopic conditions at 7.1 and $10.2 \mathrm{cpd}$ compared to mesopic conditions (Table 2).

The comparison of the CS with glare stimulus between 40 patients with photophobia and 67 without photophobia showed that the $\log C S$ of $10.2 \mathrm{cpd}$ under mesopic conditions was significantly lower in the group with subjective photophobia (Table 3).

Pearson correlation analysis revealed a statistically significant negative correlation between $\log \mathrm{CS}$ and stereopsis at all spatial frequencies except $10.2 \mathrm{cpd}$ with or without glare under mesopic conditions (Table 4). Under photopic 
Table 4. Coefficients of correlation between log contrast sensitivity and stereopsis

\begin{tabular}{lllllll}
\hline Spatial frequency (cpd) & 1.1 & 1.8 & 2.9 & 4.5 & 7.1 & 10.2 \\
\hline Mesopic without glare & $r=-0.226^{*}$ & $r=-0.255^{*}$ & $r=-0.210^{*}$ & $r=-0.233^{*}$ & $r=-0.200^{*}$ & $r=-0.181$ \\
& $p=0.019^{*}$ & $p=0.008^{*}$ & $p=0.030^{*}$ & $p=0.015^{*}$ & $p=0.039^{*}$ & $p=0.061$ \\
& $r=-0.266^{*}$ & $r=-0.310^{*}$ & $r=-0.294^{*}$ & $r=-0.353^{*}$ & $r=-0.281^{*}$ & $r=-0.187$ \\
Mesopic with glare & $p=0.006^{*}$ & $p=0.001^{*}$ & $p=0.002^{*}$ & $p=0.000^{*}$ & $p=0.003^{*}$ & $p=0.054$ \\
& $r=-0.093$ & $r=-0.192^{*}$ & $r=-0.263^{*}$ & $r=-0.234^{*}$ & $r=-0.228^{*}$ & $r=-0.210^{*}$ \\
Photopic without glare & $p=0.339$ & $p=0.047^{*}$ & $p=0.006^{*}$ & $p=0.015^{*}$ & $p=0.018^{*}$ & $p=0.030^{*}$ \\
& $r=-0.202^{*}$ & $r=-0.204^{*}$ & $r=-0.265^{*}$ & $r=-0.285^{*}$ & $r=-0.277^{*}$ & $r=-0.217^{*}$ \\
Photopic with glare & $p=0.037^{*}$ & $p=0.035^{*}$ & $p=0.006^{*}$ & $p=0.003^{*}$ & $p=0.004^{*}$ & $p=0.025^{*}$ \\
\hline
\end{tabular}

$\mathrm{cpd}=$ cycles per degree.

${ }^{*} p<0.05$.

Table 5. Coefficients of correlation between log contrast sensitivity and binocular status (fusional ability)

\begin{tabular}{lllllll}
\hline Spatial frequency (cpd) & 1.1 & 1.8 & 2.9 & 4.5 & 7.1 & 10.2 \\
\hline Mesopic without glare & $r=0.072$ & $r=0.077$ & $r=0.107$ & $r=0.120$ & $r=0.138$ & $r=0.133$ \\
& $p=0.460$ & $p=0.432$ & $p=0.273$ & $p=0.218$ & $p=0.156$ & $p=0.172$ \\
Mesopic with glare & $r=0.229^{*}$ & $r=0.298^{*}$ & $r=0.222^{*}$ & $r=0.251^{*}$ & $r=0.176$ & $r=0.116$ \\
& $p=0.018^{*}$ & $p=0.002^{*}$ & $p=0.021^{*}$ & $p=0.009^{*}$ & $p=0.070$ & $p=0.236$ \\
Photopic without glare & $r=0.042$ & $r=0.083$ & $r=0.165$ & $r=0.136$ & $r=0.140$ & $r=0.151$ \\
& $p=0.665$ & $p=0.393$ & $p=0.089$ & $p=0.161$ & $p=0.151$ & $p=0.120$ \\
Photopic with glare & $r=0.187$ & $r=0.231^{*}$ & $r=0.138$ & $r=0.174$ & $r=0.175$ & $r=0.157$ \\
& $p=0.054$ & $p=0.016^{*}$ & $p=0.155$ & $p=0.073$ & $p=0.071$ & $p=0.105$ \\
\hline
\end{tabular}

cpd $=$ cycles per degree.

$\stackrel{*}{p}<0.05$.

conditions, $\log \mathrm{CS}$ and stereopsis were significantly negatively correlated at all spatial frequencies except $1.1 \mathrm{cpd}$ without glare. Binocular status (fusional ability) was significantly positively correlated with $\log \mathrm{CS}$ at $1.8 \mathrm{cpd}$ under photopic conditions with glare, 1.1, 1.8, 2.9, and 4.5 cpd under mesopic conditions with glare (Table 5). The angle of deviation, subjective photophobia, and frequency were negatively correlated with $\log C S$ at $1.1 \mathrm{cpd}$ under mesopic conditions without glare, $10.2 \mathrm{cpd}$ under mesopic conditions with glare, and $7.1 \mathrm{cpd}$ under photopic conditions without glare.

Regarding $\Delta \log C S$ before and after glare stimulus, fusional ability was significantly negatively correlated at 4.5 cpd under mesopic conditions (correlation coefficient; $r=$ -0.197, $p=0.042$ ), subjective photophobia was significantly positively correlated at $10.2 \mathrm{cpd}$ under photopic conditions $(r=0.209, p=0.030)$, and frequency was significantly positively correlated at $2.9 \mathrm{cpd}$ under photopic conditions $(r=$ $0.244, p=0.012$ ).
Pearson correlation analysis between BCVA, SE, angle of deviation, stereopsis, binocular status, age of onset, frequency, and subjective photophobia and barring CS results revealed a statistically significant negative correlation between the age of onset and subjective photophobia $(r=$ $-0.223, p=0.021)$.

\section{Discussion}

Photophobia is a typical subjective symptom associated with IXT, requiring an objective assessment tool for its analysis. Recently, there have been several studies using the CS test in IXT, considering that CS decreases in the presence of photophobia.

Chung et al. [7] compared the preoperative CS of 58 children with IXT with that of children at 3 months after strabismus surgery as well as with 34 normal controls. The children with IXT showed significantly lower CS at low 
and intermediate spatial frequencies under mesopic conditions, and at low spatial frequencies under photopic conditions compared with normal controls. Moreover, the stimuli of glare suppressed the CS under mesopic conditions at intermediate spatial frequencies only in children with IXT, which was strongly associated with subjective symptoms of photophobia.

We previously reported differences in CS based on the surgical results (successful correction group vs. overcorrection group) in 54 patients with IXT [10]. CS under photopic conditions was significantly poorer in the overcorrection group than in the successful correction group. In addition, CS under photopic conditions was significantly correlated with subjective photophobia after surgery for IXT, suggesting its use as an objective indicator of photophobia.

In this study, there was no spatial frequency, demonstrating a significant reduction in CS by glare stimulus. This was identical to the result of our previous study, which showed no significant reduction in CS by glare stimulation at all spatial frequencies under mesopic conditions, and a tendency, with no statistical significance, of reduction in CS by glare stimulation at all spatial frequencies under photopic conditions [10]. $\triangle \mathrm{CS}$ was greater under photopic conditions at high spatial frequencies compared to mesopic conditions. This differed from the results of Chung et al. [7], who suggested that the CS was significantly reduced by glare stimulation at intermediate spatial frequency under mesopic conditions, which could possibly be due to differences in spatial frequencies according to the devices used to conduct the CS test. While Chung et al. [7] performed the CS test using the Optec 6500 vision testing system based on spatial frequencies of $1.5,3,6,12$, and $18 \mathrm{cpd}$, our study was conducted under visual angles of $6.3^{\circ}, 4^{\circ}, 2.5^{\circ}, 1.6^{\circ}, 1^{\circ}$, and $0.64^{\circ}(1.1,1.8,2.9,4.5,7.1$, and $10.2 \mathrm{cpd}$, respectively). This difference may have introduced a lower spatial frequency bias in the test results, which is equal to larger visual angles in our study, and lowered the difference between CS caused by glare stimulation.

In the correlation analysis for CS, we observed a significant correlation between CS and stereoacuity at all spatial frequencies except for $10.2 \mathrm{cpd}$ under mesopic conditions and $1.1 \mathrm{cpd}$ under photopic conditions without glare. Among the visual transmission pathways, the parvocellular pathway is responsible for color, fine texture, and high spatial frequencies, while the magnocellular pathway is responsible for luminance, motion, and low spatial frequencies [11]. Livingstone and Hubel [12] showed that stereopsis is also mediated by the magnocellular pathway. Kwon and Jung [13] used the Optec 6500 vision testing system in IXT and illustrated that the binocular CS summation ratio of low spatial frequencies under photopic conditions was negatively correlated with stereopsis, and suggested its association with the magnocellular pathway. In our study, a poor CS at 1.8, 2.9, 4.5, and $7.1 \mathrm{cpd}$ was associated with more inferior stereopsis, regardless of the presence or absence of the glare stimulus. This range of spatial frequencies (1.8 to $7.1 \mathrm{cpd}$ ) includes $3 \mathrm{cpd}$, which is a low spatial frequency that was significantly correlated with stereopsis according to Kwon and Jung [13].

A significant positive correlation between CS and fusional ability at low and intermediate spatial frequencies under mesopic conditions with glare was also revealed. The CS with glare stimuli in the group with photophobia was lower than that in the group without photophobia at a high spatial frequency (10.2 cpd) under mesopic conditions. As mentioned above, it is believed that convergence is reduced in bright outdoor areas, disrupting fusion, causing manifest strabismus, and thereby generating photophobia to eliminate diplopia or visual confusion. Consequently, CS with glare under mesopic conditions may help assess fusion and photophobia in IXT.

Chung et al. [7] reported no correlation between the magnitude of the decreased CS at intermediate spatial frequencies with the glare stimuli, and the angle of deviation, sex, age, SE, stereoacuity, binocular status, or duration of IXT, while the only factor that strongly correlated with the presence of photophobia symptoms. However, in our study, $\Delta \mathrm{CS}$ was smaller at $4.5 \mathrm{cpd}$ (intermediate spatial frequency) with better fusional ability, greater at $10.2 \mathrm{cpd}$ (high spatial frequency) with photophobia, and greater at $2.9 \mathrm{cpd}$ (intermediate spatial frequency) with higher exotropia frequency.

In the correlation analysis barring CS results, the presence of more subjective photophobia was associated with an earlier onset of IXT. Oh et al. [14] identified factors associated with photophobia by categorizing patients according to the presence or absence of photophobia based on their subjective complaints. Like our study, they reported that the IXT onset was faster in the group with photophobia $(p=0.03)$. They explained that the early onset of IXT 
involved the induction of anomalous sensory adaptation, thereby causing photophobia as a response to anomalous sensory adaptation.

The limitations of this study include its retrospective nature and small sample size. Since the subjective symptoms could not be clearly described in children, the frequency of IXT and subjective photophobia were primarily identified by their guardians, thereby influencing the accuracy of the results. Moreover, a comparison with normal controls could not be conducted. However, our study only included cases with no amblyopia and astigmatism of less than $2 \mathrm{D}$, and it is considered that the normal group under the same condition does not appeal for photophobia. Finally, we could not accurately compare our outcomes with those of other studies because the devices used to measure CS differ from those used in previous studies.

In conclusion, the CS test in this study could not represent photophobia in IXT. However, CS tended to decline when given glare, although not statistically significant, and patients with photophobia exhibited worse CS under mesopic conditions with glare. Moreover, we illustrated a significant negative correlation between stereoacuity and CS, and a significantly positive correlation between fusional ability and CS under mesopic conditions. Therefore, our study is meaningful because it presents the CS test as a tool for evaluating sensory function in IXT.

\section{Conflict of Interest}

No potential conflict of interest relevant to this article was reported.

\section{Acknowledgements}

This work was supported by the 2019 Inje University research grant.

\section{References}

1. Wang FM, Chryssanthou G. Monocular eye closure in intermittent exotropia. Arch Ophthalmol 1988;106:941-2.

2. Oh SY, Huh D, Hwang JM, Min BM. The clinical characteristics of intermittent exotropia and their relationship. $J$ Korean Ophthalmol Soc 1998;39:2797-802.

3. Manley DR. Classification of the exodeviations. In: Manley DR, editor. Symposium on horizontal ocular deviations. St Louis: Mosby; 1971. p. 128.

4. Chung ST, Legge GE. Comparing the shape of contrast sensitivity functions for normal and low vision. Invest Ophthalmol Vis Sci 2016;57:198-207.

5. Pang Y, Allen M, Robinson J, Frantz KA. Contrast sensitivity of amblyopic eyes in children with myopic anisometropia. Clin Exp Optom 2019;102:57-62.

6. Lew H, Kim CH, Yun YS, Han SH. Binocular photophobia after surgical treatment in intermittent exotropia. Optom Vis Sci 2007;84:1101-3.

7. Chung SA, Rhiu S, Han SH, Lee JB. Photophobia measurement in intermittent exotropia using the contrast sensitivity test. Graefes Arch Clin Exp Ophthalmol 2013;251:1405-11.

8. Kanazawa M, Uozato H. Relationship between absorptive lenses and contrast sensitivity in healthy young subjects with glare under photopic- and mesopic-vision conditions. Opt Rev 2013;20:282-7.

9. Savini G, Calossi A, Schiano-Lomoriello D, Barboni P. Precision and normative values of a new computerized chart for contrast sensitivity testing. Sci Rep 2019;9:16537.

10. Kim HR, Lee SJ. Comparison of contrast sensitivity based on the surgical results for intermittent exotropia. Int J Ophthalmol 2019;12:1725-30.

11. Cheng A, Eysel UT, Vidyasagar TR. The role of the magnocellular pathway in serial deployment of visual attention. Eur J Neurosci 2004;20:2188-92.

12. Livingstone MS, Hubel DH. Psychophysical evidence for separate channels for the perception of form, color, movement, and depth. J Neurosci 1987;7:3416-68.

13. Kwon JM, Jung JH. Subnormal binocular contrast sensitivity summation in patients with intermittent exotropia. $J$ Korean Med Sci 2018;33:e222.

14. Oh BL, Suh SY, Choung HK, Kim SJ. Squinting and photophobia in intermittent exotropia. Optom Vis Sci 2014;91:5339. 